\title{
Arthroscopic Foveal Repair of the Triangular Fibrocartilage Complex
}

\author{
Andrea Atzei, MD ${ }^{1}$ Riccardo Luchetti, MD ${ }^{2}$ Federica Braidotti, $\mathrm{PT}^{1}$ \\ 1 Fenice Hand Surgery and Rehabilitation Team, Treviso, \\ Pordenone, Italy \\ 2 Rimini Hand and Rehabilitation Center, Rimini, Italy \\ Address for correspondence Andrea Atzei, MD, Fenice Hand Surgery \\ and Rehabilitation Team, Viale Louis Pasteur, 6/A, 31100 Treviso, Italy \\ (e-mail: andreatzei@gmail.com).
}

J Wrist Surg 2015;4:22-30.

\begin{abstract}
Background Foveal disruption of the triangular fibrocartilage complex (TFCC) is associated with distal radioulnar joint (DRUJ) instability. TFCC fixation onto the fovea is the suitable treatment, which is not achieved by conventional arthroscopic techniques. We describe an all-inside arthroscopic technique that uses a suture anchor through distal DRUJ arthroscopy for foveal repair of the TFCC.

Materials and Methods Forty-eight patients with TFCC foveal tear and DRU] instability were selected according to the Atzei-European Wrist Arthroscopy Society (EWAS) algorithm of treatment. Retrospective evaluation included pain, DRUJ instability, range of motion (ROM), grip strength, Modified Mayo Wrist Score (MMWS), and the Disabilities of the Arm, Shoulder, and Hand (DASH) Score.

Description of Technique DRUJ arthroscopy was performed to débride the TFCC and the foveal area. Under arthroscopic guidance, a suture anchor was inserted via the distal foveal portal to repair the TFCC onto the fovea. Sutures were tied on the radiocarpal surface of the TFCC. Postoperative immobilization of forearm rotation was maintained for 4 weeks. Heavy tasks were allowed after 3 months.

Results After a mean follow-up of 33 months, pain improved significantly but remained moderate in four patients, severe in one. DRUJ instability resolved in 44

Keywords

- triangular fibrocartilage complex

- TFCC

- foveal repair

- arthroscopy

- DRUJ instability patients. Wrist ROM increased. Grip strength, MMWS, and DASH score improved significantly. Excellent and good MMWS equaled 83.3\%. Forty-one patients (85.5\%) resumed previous work and sport activities. As a postoperative complication, five patients experienced neuroapraxia of the dorsal sensory branch of the ulnar nerve (DSBUN) with full spontaneous recovery.

Conclusions With appropriate indications and patient selection, arthroscopic foveal repair of the TFCC may restore DRUJ stability and provide satisfactory results without significant complications.
\end{abstract}

Traumatic disruption of the Triangular Fibrocartilage Complex (TFCC) is a common cause of ulnar-sided wrist pain and is often associated with decreased grip strength and impaired function. The Palmer classification ${ }^{1}$ defines traumatic tears involving the ulnar periphery of the TFCC as type 1-B. However, according to the magnitude and direction of the traumatic force, the TFCC may rupture in a variable manner, and the ulnar styloid may also fracture, producing different subtypes of type 1-B tears. Treatment options are variable, depending on the tear's features. We proposed a treatmentoriented classification system ${ }^{2}$ that subdivided type 1-B TFCC tears into five classes, allowing differentiation between distal and proximal lesions (i.e., involving the foveal insertions of the TFCC), as well as reparable or irreparable lesions (-Table 1). The European Wrist Arthroscopy Society (EWAS) endorsed the classification. Recently, it was updated
Copyright (c) 2015 by Thieme Medical Publishers, Inc., 333 Seventh Avenue, New York, NY 10001, USA. Tel: +1(212) 584-4662. 
Arthroscopic Foveal Repair of the TFCC Atzei et al. 23

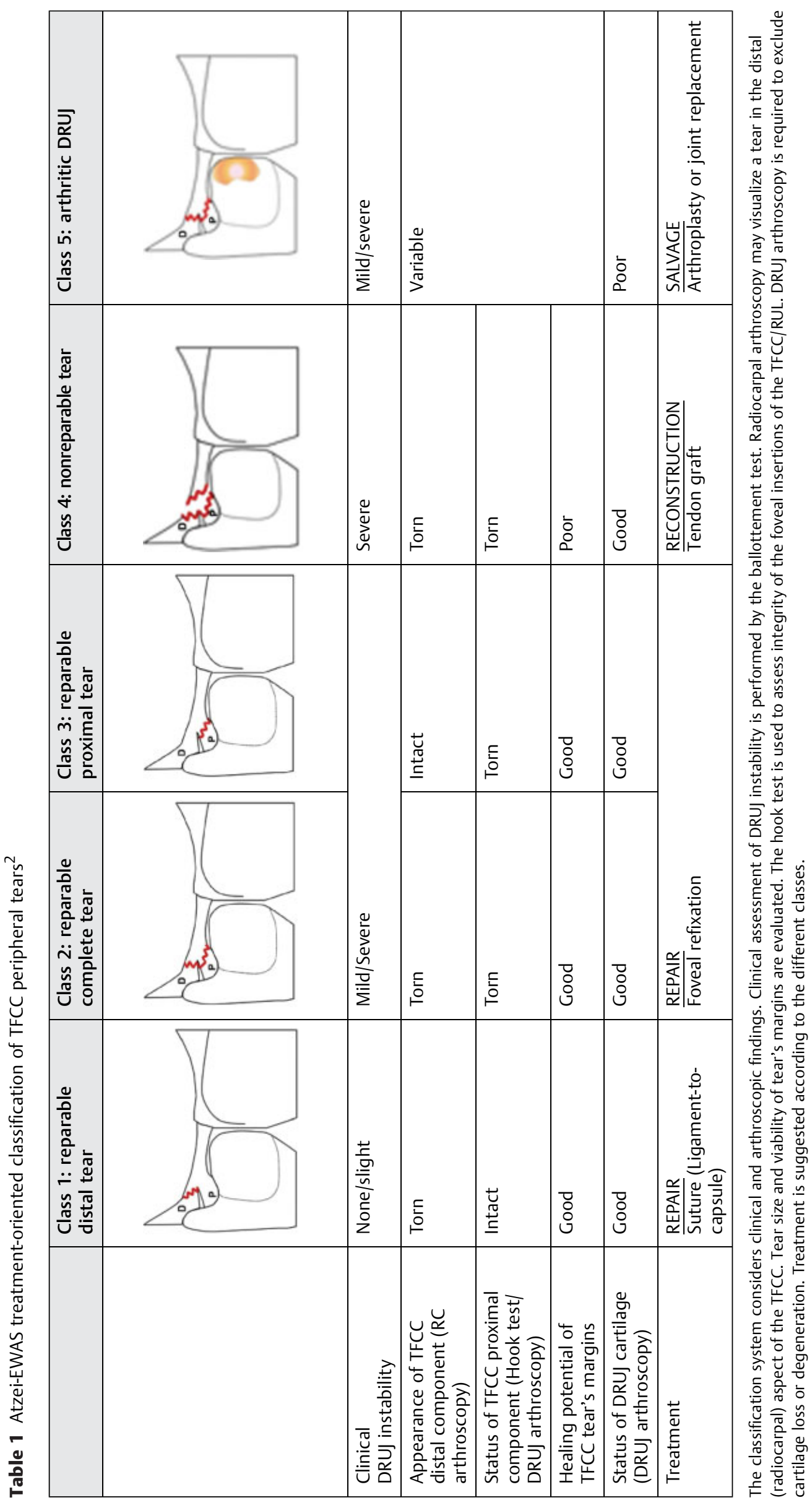


by adding radiographic assessment of ulnar styloid fracture. ${ }^{3}$ When deciding the appropriate treatment for repairable 1-B TFCC tears, the most important parameter to consider is the interruption of the foveal insertion of the TFCC. Foveal fibers represent the ulnar origin of the radioulnar ligament (RUL), and its rupture causes not only pain but also distal radioulnar joint (DRUJ) instability. ${ }^{4,5}$ A review of the recent literature on TFCC repair confirms that DRUJ instability is the most common cause of unsatisfactory results and reoperations. ${ }^{4,6}$ This finding is related either to a failure to diagnose DRUJ instability or to an inadequate TFCC repair. ${ }^{6}$ Routine preoperative assessment of DRUJ instability with the ballottement test, then repeated with the patient under anesthesia, decreases the risk of misdiagnosis. ${ }^{7}$ DRUJ arthroscopy allows visualization of the foveal origins of the TFCC, ${ }^{8-10}$ and the "hook test" is a reliable method to assess its competence. ${ }^{2,11}$ Both open and arthroscopic techniques have been described for repairing TFCC tears and improving DRUJ stability. ${ }^{4,11-35}$ An arthroscopic approach minimizes the soft tissue trauma and provides an improved diagnostic accuracy. Conventional arthroscopic techniques of TFCC repair consist of a simple suturing of the tear to the dorsal ulnar joint capsule and do not address the foveal insertion. Proper repair of foveal disruptions of the TFCC requires reinsertion of the deep fibers onto the ulnar fovea, which can be performed with transosseous sutures by either open or arthroscopic approach. ${ }^{28}$ We developed an arthroscopic-assisted technique that uses suture-anchors for a foveal repair of the TFCC. ${ }^{11}$ Technical refinements of DRUJ arthroscopy have enabled further modification of the original technique into an all-inside arthroscopic procedure. We describe technical details of the technique and report midterm results.

\section{Patients and Methods}

We performed a retrospective analysis of the medical records of 159 patients who underwent wrist arthroscopy for peripheral TFCC tear between 2009 and 2013. Foveal rupture with DRUJ instability was diagnosed in 118 patients (74.21\%). In this group, there were 84 cases (71.18\%) of complete tear (Class 2), and 18 cases (15.25\%) of isolated rupture of the foveal fibers of the TFCC (Class 3). Associated lesions were observed in 27 cases. Forty-one cases (26.79\%) that were diagnosed as distal TFCC tear with intact foveal insertions (Class 1 ) and 16 cases (13.57\%) in which the tear was not repairable with sutures (Class 4 ), as well as the cases with associated lesions, were excluded from the study. Thus, a total of 75 patients were eligible for review. Forty-eight patients (64\%) were available for follow-up evaluation. There were 28 male and 20 female patients. The dominant hand was involved in 27 patients. The average age at surgery was 34 years (range 17 to 54 years). The mean period of time from the injury until the operation was 11 months (range 5 to 19 months). Before surgery, 35 patients (73\%) participated in vigorous physical activity (either working or recreational), and 15 (31\%) were high-level athletes. The mechanism of injury included an acute event (fall or violent twisting of the wrist) in all cases. The average follow-up was 33 months (range 6 to 52 months). The clinical outcomes were evaluated using a visual analog scale (VAS) for pain at rest and during activity, the Modified Mayo Wrist Score, and the Disability of the Arm, Shoulder, and Hand (DASH) score. Recovery of DRUJ stability was defined by the absence of a painful click or crepitus during forearm rotation and by the presence of "firm resistance" at the endpoint of DRUJ translation during the ballottement test. The wrist range of motion (ROM) and grip strength were measured with a standard goniometer and a Jamar dynamometer. The results obtained were reported as percent of the contralateral side. Paired $t$-tests were conducted to analyze differences in clinical and functional measures before and after foveal repair. The obtained data were expressed as mean \pm standard deviation. The level of significance was set at $p<0.05$.

\section{Surgical Technique}

Patients with ulnar-sided wrist pain and impaired function due to a clinically unstable DRUJ who show no improvement after a minimum 3 months trial of conservative treatment undergo wrist arthroscopy to rule out a foveal tear of the TFCC. High-demand patients or patients with grossly unstable DRUJ may undergo earlier treatment. Patient selection for arthroscopic-assisted foveal repair is based mainly on intraoperative arthroscopic findings, according to the Atzei-EWAS classification $^{2}$ (- Table 1). Standard radiocarpal (RC) portals are used to visualize and probe a Class 2 lesion, which is visible at the dorsoulnar side of the TFCC (-Fig. 1). In a Class 3 lesion, the tear is not visible on the RC aspect of the TFCC, since only the foveal fibers are interrupted; thus, the tear is assessed via DRUJ arthroscopy. A positive trampoline test with loss of the normal buoyancy of the TFCC indicates the presence of a tear of the TFCC. ${ }^{19}$ Competence of the foveal insertions is assessed using the hook test ${ }^{2,3}$ : traction is applied with a probe on the ulnar margin of the TFCC. If the foveal fibers are torn, the articular disk displaces toward the center of the RC joint (-Fig. 2). DRUJ arthroscopy can be used to visualize the lacerated fibers and the "vacant fovea"3,20 and is highly correlated with a positive hook test. (Atzei A. Presented at 11th Triennial congress of the IFSSH, 2010). A positive hook test is reliable indicator of foveal rupture and DRUJ arthroscopy is no longer performed for
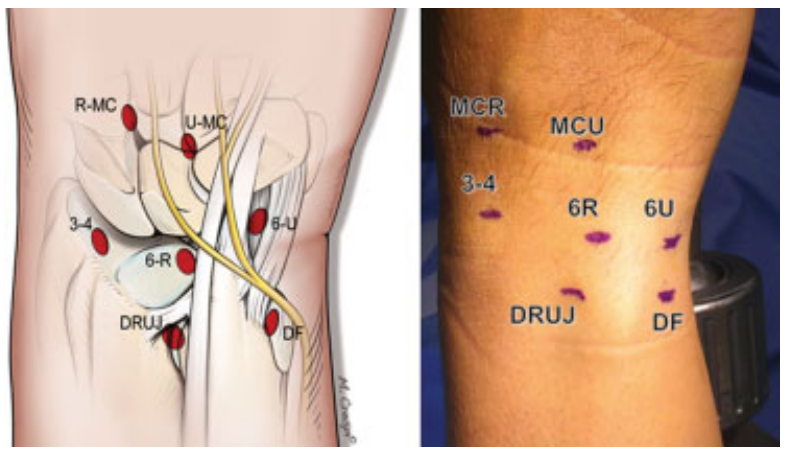

Fig. 1 Standard portals for radiocarpal (3-4; 6R; 6U), midcarpal (MCR; $M C U)$, and DRUJ (DRUJ; DF) arthroscopy. The dorsal sensory branch of the ulnar nerve (DSBUN) is outlined. 


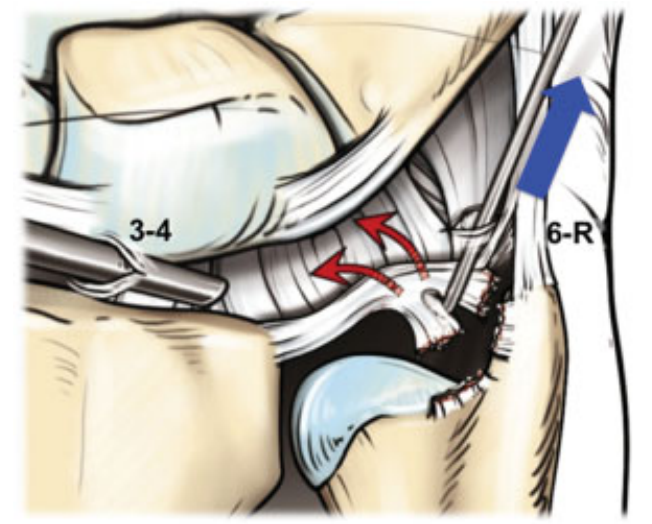

Fig. 2 The hook test evaluates tautness of the foveal insertions of the proximal-TFCC through radiocarpal arthroscopy (scope 3-4). The probe is inserted through the $6 \mathrm{R}$ portal in the prestyloid recess and applies traction to the TFCC. When the foveal fibers are lacerated, the TFCC can be displaced toward the center of the RC joint. The test is considered positive.

confirmatory purposes. Classes 2 and 3 of the Atzei-EWAS classification $^{2}$ are candidates for an arthroscopic foveal repair. The suitability of a Class 4 tear for repair is judged based on the tear's size and the quality of its edges. A contraindication to repair would include suturing under tension due to large tears or repair of chronic tears with degenerated or necrotic edges that cannot be débrided to healthy margins. Foveal repair is also contraindicated in the presence of cartilage loss or degeneration at the DRUJ (Class 5), chronic Essex-Lopresti injury, previous soft tissue infection, osteomyelitis, or severe osteoporosis of the ulnar head. Carpal chondromalacia, secondary to chronic ulnar impaction, and dysplasia of the sigmoid notch are relative contraindications. The technique is performed with a standard wrist arthroscopy setup using finger traps and a wrist traction tower, with 10-12 lbs (4.5$5.4 \mathrm{~kg}$ ) of longitudinal traction. ${ }^{37}$ Joint distension with saline is usually not required, and the use of the dry technique $e^{37,38}$ is advisable. A 2.7-mm arthroscope is used routinely. A 1.9-mm arthroscope may be necessary for smaller wrists. The scope is introduced through the 3-4 portal. The $6 \mathrm{R}$ and $6 \mathrm{U}$ portals are created for probe and instrument introduction and then for suture retrieval and knotting. Class 2 lesions are débrided through the RC portals. Class 3 lesions are débrided through DRU arthroscopy. A direct foveal (DF) portal for exploring the DRUJ is created midway between the standard proximal DRUJ portal and the distal DRUJ portal ${ }^{8}$ (-Fig. 3). The DF portal is the working portal for tear débridement and suture anchor fixation (-Fig. 4a,b). ${ }^{11}$ As a reference for portal placement, a 22 -gauge needle is introduced $\sim 1 \mathrm{~cm}$ proximal to the $6 \mathrm{U}$ portal, just palmar to the ulnar styloid. The DF portal is created with the forearm fully supinated and with careful blunt soft tissue spreading, thus minimizing the risk of damage to the dorsal sensory branch of the ulnar nerve (DSBUN). Full forearm supination also allows easier access to the foveal area via the DF portal for tear débridement and for suture anchor drilling and insertion (-Fig. 5a,b). A small (2.0-mm) power shaver and/or a curette are used to débride the torn foveal fibers and to create a bleeding surface on the

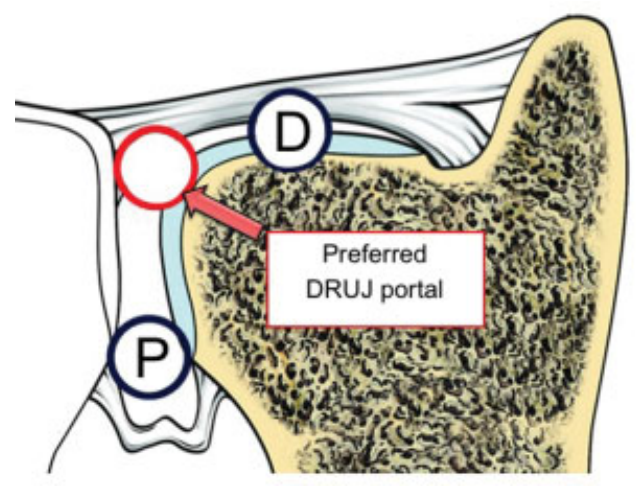

Fig. 3 Schematic drawing of the dorsal DRUJ portals. The authors' preferred DRUJ portal is created midway between the standard proximal DRUJ portal (P) and the distal DRUJ portal (D).

fovea ulnaris by exposing the cancellous bone ( - Fig. $\mathbf{6}$ ). Then a suture anchor can be easily inserted onto the foveal area (-Fig. 7). We prefer to use a self-tapping suture anchor loaded with a pair of sutures (four strands), to repair each limb of the RUL. When the suture anchor handle is removed, the four sutures exit the DRUJ from the DF portal (-Fig. 8). One suture is palmar and the other one more dorsal, each suture having a radial strand and an ulnar one. The radial strand of each suture is loaded into the tip of a 25-gauge hypodermic or Tuohy needle and, with the scope still in the DRUJ portal, is introduced into the DF portal. Aiming upwards, the needle pierces the palmar RUL and next the dorsal RUL, protruding into the RC joint ( $\mathbf{- F i g . 9}$ ). Then the scope is shifted to the 3-4 portal to confirm correct needle placement. The sutures are retrieved through the $6 \mathrm{U}$ portal (-Fig. 10). The same procedure is repeated with the ulnar strand of each suture, which protrudes into the prestyloid recess (-Fig. 11). Then they are retrieved through the $6 \mathrm{U}$ portal. At the end of the procedure, all sutures exiting through the $6 \mathrm{U}$ portal are cleared from any interposed soft tissue (-Fig. 12). Using a knot-pusher through the 6-U portal, a sliding knot is tied under arthroscopic guidance (scope in the 3-4 portal) and placed in the presyloid recess (-Fig. 13). During knot tying, the forearm is in neutral rotation and

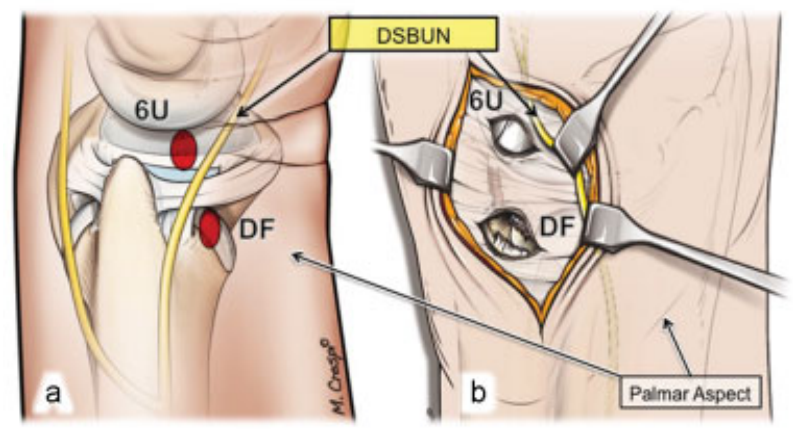

Fig. 4a,b (a) The direct foveal (DF) portal is located $\sim 1 \mathrm{~cm}$ proximal to the $6 \mathrm{U}$ portal, on the medial aspect of the wrist. (b) For less experienced surgeons, or in small wrists, it may be advisable to use a 2- to $2.5-\mathrm{cm}$ miniopen approach, just proximal to the $6 \mathrm{U}$ portal, which allows identification and protection of the DSBUN with two small Ragnell retractors. 


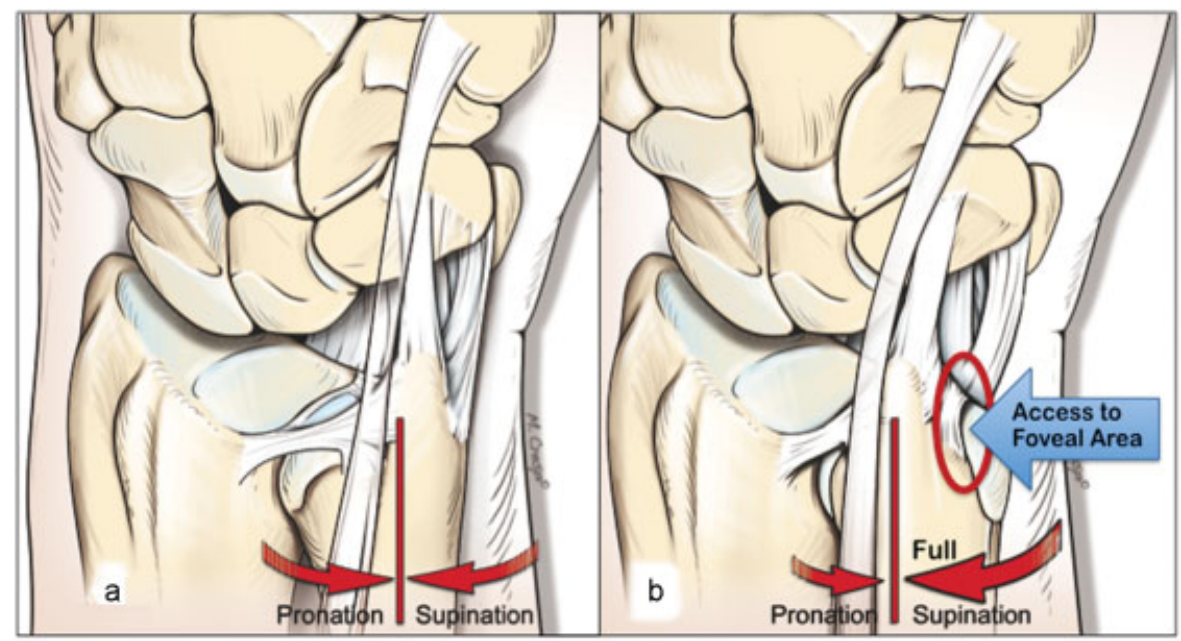

Fig. 5a,b Artist's rendering demonstrates the modification of the anatomical relationships of the ulnar wrist from (a) neutral prono-supination, as in the standard arthroscopic setup, to (b) full forearm supination, which is the recommended position to create the DF portal. In full forearm supination, the ulnar styloid and the ECU tendon displace dorsally and allow the foveal area of the distal ulna to move more superficially and become subcutaneous, so that it can be easily reached subcutaneously for débridement and for suture anchor drilling and insertion.

wrist traction is released. When necessary, the assistant holds the ulnar head in a reduced position.

Rehabilitation. After an arthroscopic foveal repair of the TFCC, the forearm is placed in a long-arm cast in neutral rotation or slight $\left(\sim 45^{\circ}\right)$ supination. After 1 week, it is changed to a Münster-type thermoplastic splint, which restricts forearm rotation and allows limited elbow flexion/extension. The splint is maintained for the following 3 weeks. Then proprioceptive rehabilitation with isometric

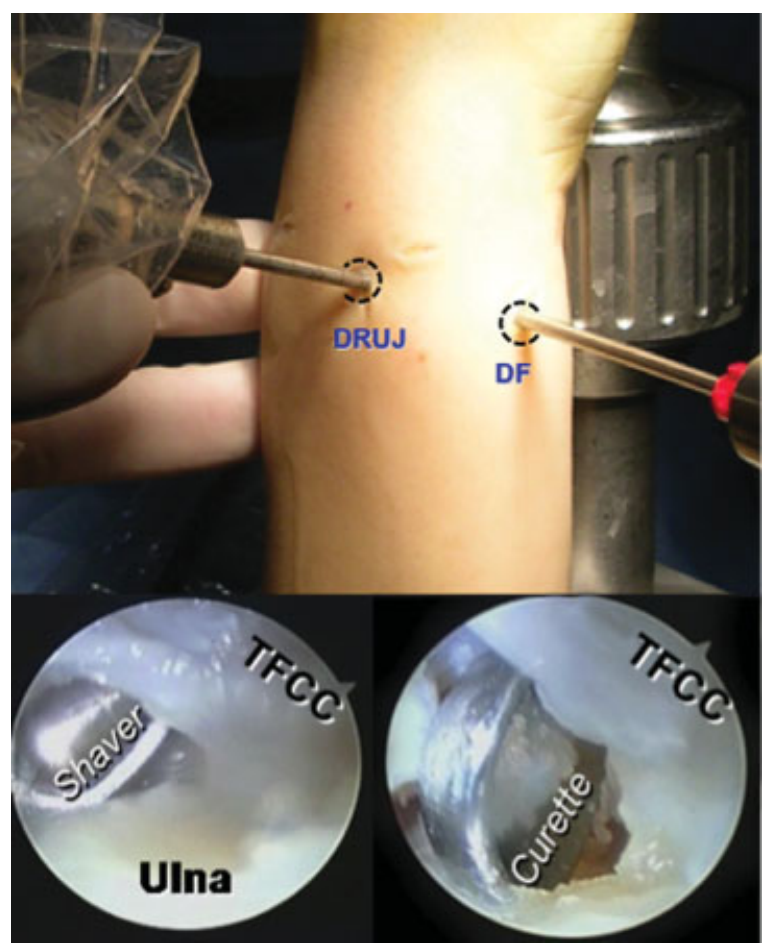

Fig. 6 DRUJ arthroscopy of a right wrist (the scope in DRUJ portal). The DF portal allows access to the foveal area. (a) A small shaver is introduced to débride scarring tissue, granulation tissue and unstable remnants of the foveal fibers of the TFCC. (b) A curette creates a bleeding surface onto the foveal area for optimal healing of the repair. exercises, co-contraction of the pronator quadratus and ECU and flexion/extension of the wrist starts at 4 weeks. Six weeks postoperatively, progressive forearm rotation is allowed and the patient can remove the splint during the day. Full ROM and resisted wrist and hand strengthening exercises are not permitted until the patient recovers consistent proprioceptive control. Daily activities can be resumed as soon as 2 months postoperatively. However, sport and heavy work tasks usually require more than

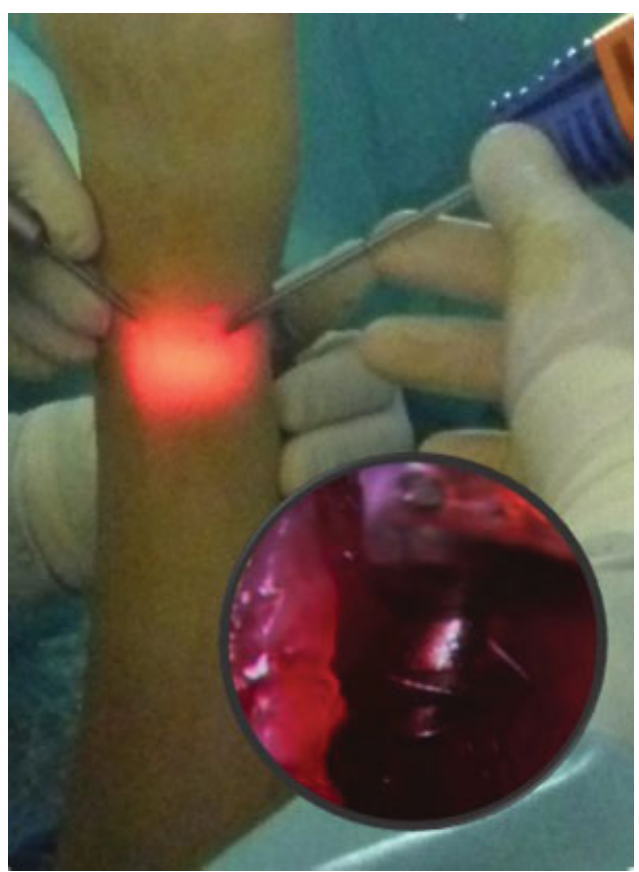

Fig. 7 Arthroscopic foveal repair with suture anchor (right wrist). After adequate débridement, the suture anchor is introduced portal into the fovea through the DF portal, under DRUJ arthroscopic guidance (scope in DRUJ). 


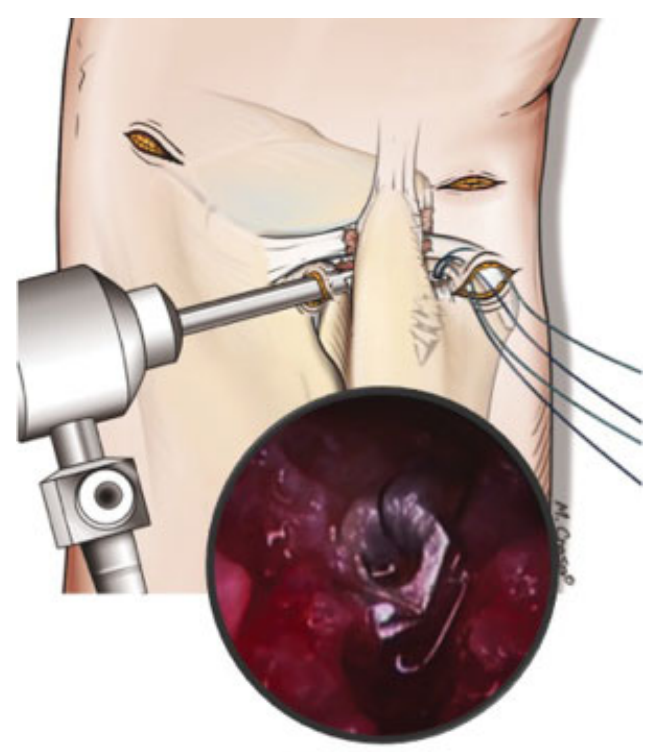

Fig. 8 DRUJ arthroscopy view after suture anchor insertion: the sutures are visible exiting through the DF portal, as demonstrated also by the artist's rendering.

3 months, according to the recovery of forearm muscle strength and endurance.

\section{Results}

There was a statistically significant improvement in VAS score for pain at rest, with a preoperative mean of $3 \pm 2$ as compared with a postoperative mean of $1 \pm 1$, as well as in pain during activity, with preoperative and postoperative mean of $8 \pm 3$ and $3 \pm 3$, respectively. However, not all cases demonstrated the same degree of improvement: pain at rest remained unchanged in one case, and pain during activity remained unchanged in two cases and worsened in one case. Moderate pain persisted in four patients (8.3\%) and severe pain in one (2\%). The mean preoperative MMWS improved

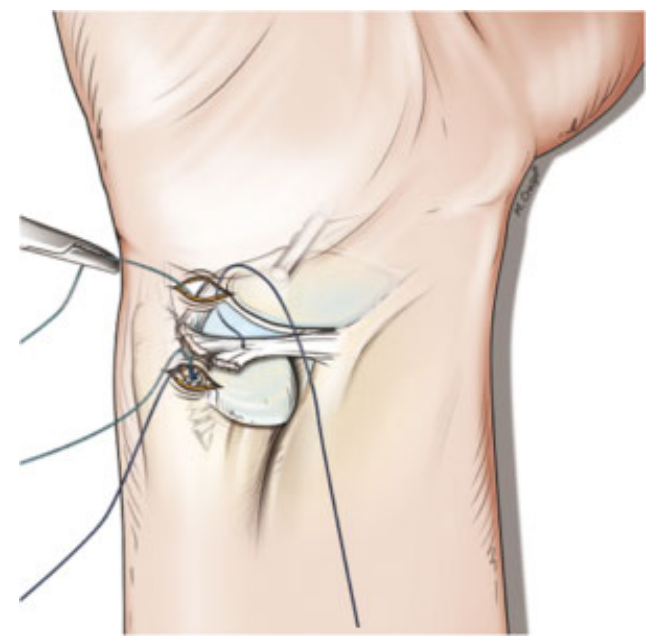

Fig. 10 A grasper is used to retrieve the sutures through the $6 \mathrm{U}$ portal.

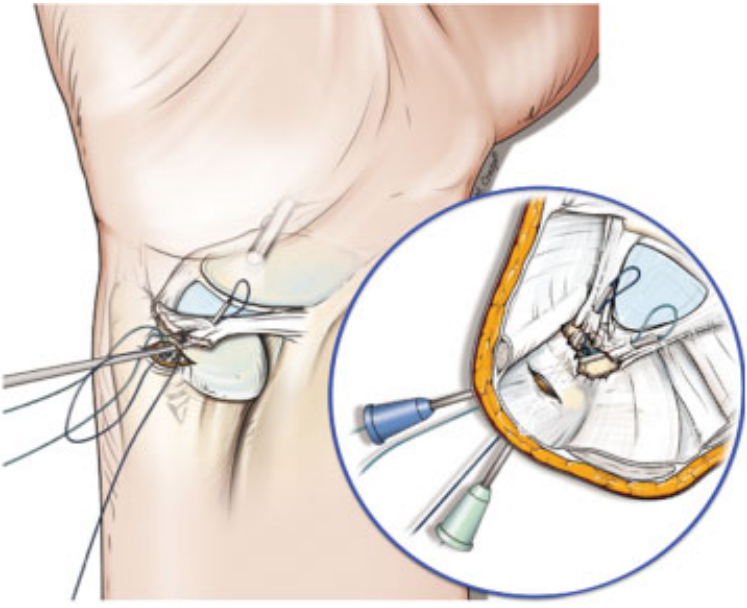

Fig. 9 The radial strand of the palmar suture (green) is loaded into the tip of a 25-gauge hypodermic or Tuohy needle. Under arthroscopic guidance, the needle is introduced into the DF portal through the TFCC to exit in the RCJ. Aiming upward, the needle pierces the TFCC close to its palmar edge, to grasp the palmar RUL and the origin of ulnocarpal ligaments. Then the radial thread of the dorsal suture (blue) is introduced through the DF portal to pierce the dorsal edge of the TFCC, to grasp the dorsal RUL. The scope is then moved to the 3-4 portal to ensure correct suture placement.

from a preoperative value of $48 \pm 13$ to a postoperative value of $87 \pm 17$, reaching statistical significance. There were 35 excellent scores (72,9\%), 5 good (10,4\%), 6 fair (12,5\%) and 2 poor (4,2\%). Excellent and good scores accounted for a total of $83.3 \%$. Forty-one patients $(85.5 \%)$ resumed their previous work and sport activities, 5 patients (10.4\%) had restricted function, and $2(4.2 \%)$ retired from work but resumed recreational activities. The DASH score improved significantly from a mean preoperative value of $42 \pm 20$ to a mean postoperative value of $15 \pm 15$. DRUJ instability resolved in 44 patients (91.7\%). Four patients (8.3\%) showed persistence of a "soft endpoint" at the ballottement test, and two of them also complained of the persistence of a painful click during forearm rotation. The mean postoperative ROM of flexion/extension of the wrist increased from $92.5 \% \pm 6 \%$

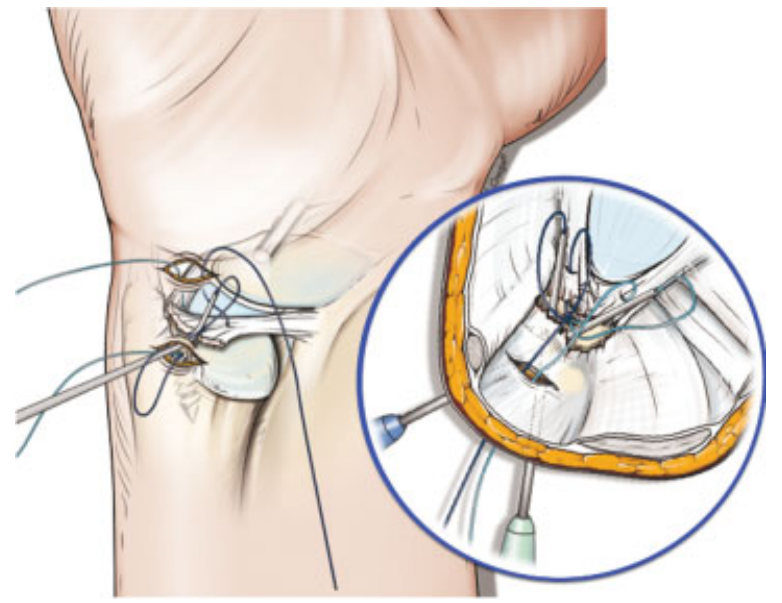

Fig. 11 The needle is loaded with the ulnar thread of each suture and introduced from the DF portal through the prestyloid recess to pierce the ulnarmost edges of the tear. 


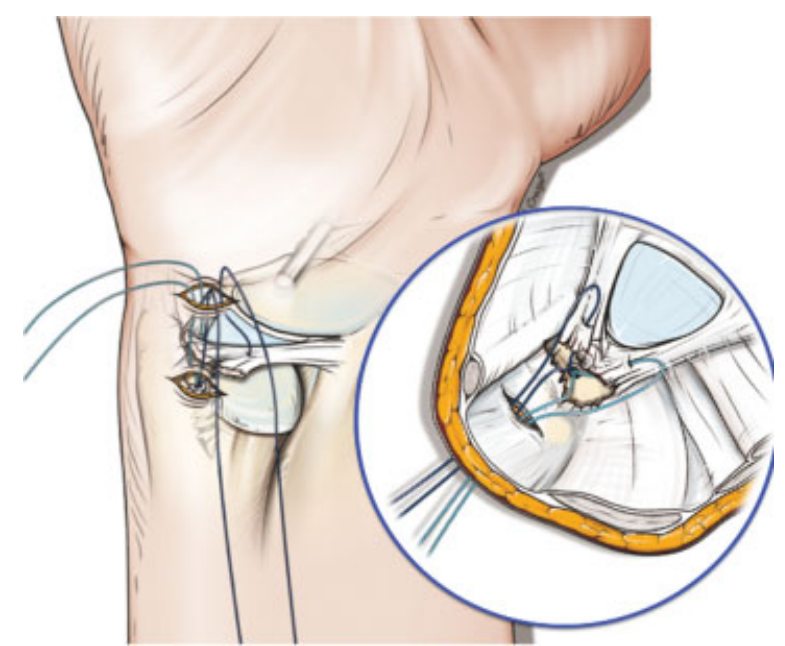

Fig. 12 At the end of the procedure, all sutures arising from the suture anchor inserted into the fovea, have pierced both edges of the TFCC on its palmar (green) and dorsal (blue) side and exited from the RC joint through the $6 \mathrm{U}$ portal.

to $98.2 \% \pm 7 \%$. The ROM of pronation/supination of the forearm increased from $92.5 \% \pm 13 \%$ to $99.5 \% \pm 17 \%$. However, no statistically significant difference was detected between the preoperative and postoperative values. The grip strength improved significantly from $92.7 \% \pm 19 \%$ to 103 , $6 \% \pm 16 \%$ postoperatively $(p<0.05)$. The only complication recorded was neurapraxia of the DSBUN, which occurred in five patients (10.4\%), all female patients with relatively small wrists. This neurapraxia recovered uneventfully in a mean of 3.7 months and did not influence the end result at follow-up. No complications were detected related to suture knot irritation.

\section{Discussion}

Palmer 1-B tears are defined as tears of the peripheral TFCC and may be associated with a disruption of the foveal insertions, where mainly the RUL originates. A foveal detachment entails greater functional impairment than simple ulnar wrist pain, as it may be associated with DRUJ instability. ${ }^{5,9}$ Therefore, repair of the TFCC to the fovea is the rational approach to relieve pain and restore good DRUJ function.

Hermansdorfer and Kleinman ${ }^{19}$ were the first to report the results of open TFCC repair performed through drill holes in the distal ulna, showing good results in 11 patients with a follow-up greater than one year.

Chou et al ${ }^{15}$ used a suture anchor to repair the TFCC to the foveal area and demonstrated that clinical results were comparable to those of transosseous repair, although the technique was remarkably easier and quicker to perform. However, the outcome evaluation of these series focused only on pain relief and restoration of ROM and grip strength; DRUJ instability was not considered in the preoperative and postoperative clinical evaluation.

The issue of DRUJ instability was addressed specifically by Nakamura et al, ${ }^{27}$ who proposed an original open technique to

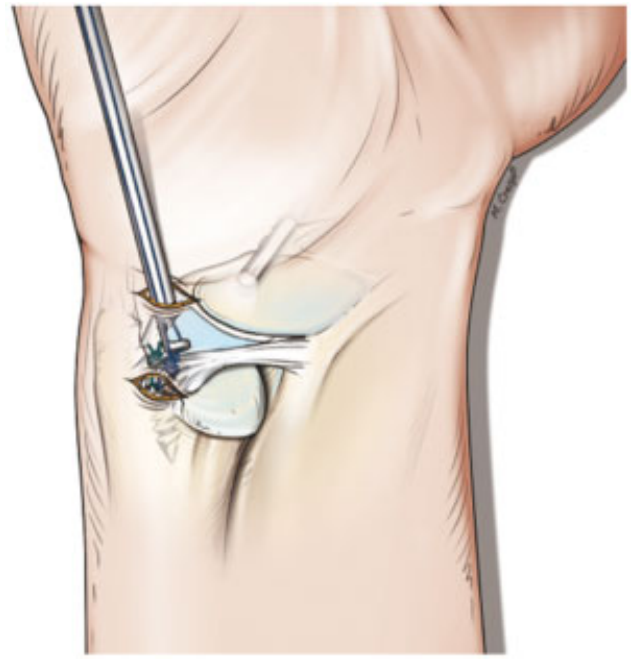

Fig. 13 Green and blue sutures are tied under arthroscopic giudance using a sliding knot and a small knot pusher. Wrist traction should be released and the ulnar head reduced with the forearm in neutral rotation.

repair TFCC foveal tears associated with DRUJ instability, validating the anatomic and functional equivalence of the proximal part of the peripheral TFCC with the RUL. Using an open approach through the floor of the extensor carpi ulnaris (ECU) tendon sheath, the tear was repaired with a transosseous three-dimensional double mattress technique that sutured the disrupted RUL to the fovea. The outcome of 66 wrists treated with this technique was evaluated after an average follow-up of 3 years. ${ }^{28}$ Pain resolved in 60 wrists (91\%) and remained severe in 2 (3\%). There was no postoperative DRUJ instability in 56 wrists (84.9\%), and moderate to severe DRUJ instability persisted in 4 wrists (6\%). The clinical results, evaluated with a specific DRUJ evaluating system score, were 56 excellent (85\%), 6 good (9\%), 2 fair (3\%), and 2 poor (3\%). Excellent and good results accounted for a total of $94 \%$.

In 2010 Moritomo et al $^{26}$ reported the clinical results of 15 patients with TFCC foveal avulsion who were treated with open repair. Pain resolved in 6 patients (40\%), mild or moderate pain persisted in 9 (60\%). The average follow-up grip strength was $90 \%$ of the unaffected side. A total of 11 patients (73.3\%) had no DRUJ instability, and 4 (26.7\%) had mild or moderate DRUJ instability. MMWS results were excellent in nine patients (60\%), good in four $(26.7 \%)$, and fair in two (13.3\%). Excellent and good results accounted for a total of $86.7 \%$.

In 2008 we introduced a less invasive arthroscopic technique of foveal repair that used a suture anchor though a mini-open approach over the $6 U$ and DF portals and reported good results in a preliminary series of 18 patients evaluated after a mean follow-up of 18 months. ${ }^{11}$ The technique underwent further refinements following increased technical proficiency on DRUJ arthroscopy, especially in relation to the safer creation and use of $6 U$ and DF portals. Thus, the technique is currently performed as a combined RC and DRU all-arthroscopic procedure.

Iwasaki and Minami ${ }^{21}$ described an arthroscopically assisted technique that made a $2.9-\mathrm{mm}$ osseous tunnel from the 
ulnar neck to the foveal region, using a targeting device using a cannulated drill under fluoroscopic guidance. They showed that bone bleeding from the transosseous tunnel could enhance adhesion of the avulsed TFCC to the foveal area. Twelve patients were observed for a mean of 30 months after arthroscopic foveal repair. ${ }^{22}$ Six patients (50\%) showed no pain, five (41.4\%) had mild pain, and one (8.3\%) had moderate pain; DRUJ instability was eliminated in all patients. The mean postoperative MMWS was 93, and the results were excellent in eight patients (66.7\%) in this series and good in four (33.3\%).

Nakamura et $\mathrm{al}^{28}$ introduced an arthroscopically assisted outside-in technique that made 2 separate $1.2-\mathrm{mm}$ osseous tunnels using a specially designed targeting device through the RC joint. Clinical results of 24 wrists were reported with an average follow-up of 3.5 years. Fifteen wrists (62.5\%) had no pain, two $(8.3 \%)$ had severe pain, and four (16.6\%) had recurrent pain. A total of $17(70.8 \%)$ of these wrists had no postoperative DRUJ instability, whereas 7 (29.2\%) had moderate to severe DRUJ instability. Final clinical results were excellent in 13 patients (54.2\%), good in 3 (12.6\%), fair in 4 (16.6\%), and poor in 4 (16.6\%). Excellent and good scores accounted for a total of $66.8 \%$.

Shinohara et $\mathrm{al}^{31}$ modified Nakamura's technique by placing the osseous tunnels more precisely at the foveal using DRUJ arthroscopy instead of RC arthroscopy. Eleven patients were evaluated after a mean follow-up of 30 months. DRUJ instability was eliminated in nine patients (81.8\%), and mild DRUJ instability persisted in two patients (18.2\%). Pain resolved completely in seven patients (66.6\%), mild pain during activity persisted in four patients (36.4\%). Grip strength improved significantly. The MMWS was excellent in seven patients (63.6\%), good in three $(27.3 \%)$, and fair in one $(9.1 \%)$. Excellent and good scores accounted for a total of $91.9 \%$.

In a recent paper, Luchetti et $\mathrm{al}^{38}$ showed that both open and arthroscopic techniques are equally valuable in repairing foveal TFCC tears and restoring DRUJ stability, though with significantly better subjective outcomes for the arthroscopic group.

The results of the present series confirm those of Kim et $\mathrm{al}^{23}$ who reported very similar clinical results using a suture anchor technique of foveal repair through DRUJ arthroscopy and DF portal. In a series of 15 patients followed up for a mean of 29 months, DRUJ instability disappeared in all cases. The wrist ROM did not show a statistically significant change after surgery. The grip power improved significantly. The MMWS was excellent in $10(66.6 \%)$ cases, good in $2(13.4 \%)$, and fair in 3 (20\%). Excellent and good scores accounted for a total of $80 \%$. The mean DASH score showed statistically significant improvement. There were two cases of ulnar nerve irritation, which improved immediately after surgery, and one case of knot irritation associated with ECU tendinitis. These very favorable results were obtained regardless of the presence of a high degree of positive ulnar variance (UV) (mean $1.7 \pm 1.0$ $\mathrm{mm}$ ). As in similar studies on DRUJ instability, limitations of this study are related to the lack of precise pre- and postoperative assessment.

\section{Note}

Work was done at Fenice Hand Surgery and Rehabilitation Team, Treviso, Pordenone, Italy

Conflict of Interest

None

\section{References}

1 Palmer AK. Triangular fibrocartilage complex lesions: a classification. J Hand Surg Am 1989;14(4):594-606

2 Atzei A. New trends in arthroscopic management of type 1-B TFCC injuries with DRUJ instability. J Hand Surg Eur Vol 2009;34(5): 582-591

3 Atzei A, Luchetti R. Foveal TFCC tear classification and treatment. Hand Clin 2011;27(3):263-272

4 Estrella EP, Hung LK, Ho PC, Tse WL. Arthroscopic repair of triangular fibrocartilage complex tears. Arthroscopy 2007;23(7): 729-737, 737.e1

5 Lindau T, Adlercreutz C, Aspenberg P. Peripheral tears of the triangular fibrocartilage complex cause distal radioulnar joint instability after distal radial fractures. J Hand Surg Am 2000; 25(3):464-468

6 Anderson ML, Larson AN, Moran SL, Cooney WP, Amrami KK, Berger RA. Clinical comparison of arthroscopic versus open repair of triangular fibrocartilage complex tears. J Hand Surg Am 2008; 33(5):675-682

7 Moriya T, Aoki M, Iba K, Ozasa Y, Wada T, Yamashita T. Effect of triangular ligament tears on distal radioulnar joint instability and evaluation of three clinical tests: a biomechanical study. J Hand Surg Eur Vol 2009;34(2):219-223

8 Atzei A, Luchetti R. DRUJ arthroscopy. In: Slutsky D, ed. Operative Orthopaedics of the Upper Extremity. New York, NY: McGraw-Hill; 2014:407-418

9 Haugstvedt JR, Berger RA, Nakamura T, Neale P, Berglund L, An KN. Relative contributions of the ulnar attachments of the triangular fibrocartilage complex to the dynamic stability of the distal radioulnar joint. J Hand Surg Am 2006;31(3):445-451

10 Slutsky DJ. Distal radioulnar joint arthroscopy and the volar ulnar portal. Tech Hand Up Extrem Surg 2007;11(1):38-44

11 Atzei A, Rizzo A, Luchetti R, Fairplay T. Arthroscopic foveal repair of triangular fibrocartilage complex peripheral lesion with distal radioulnar joint instability. Tech Hand Up Extrem Surg 2008;12(4): 226-235

12 Badia A, Jiménez A. Arthroscopic repair of peripheral triangular fibrocartilage complex tears with suture welding: a technical report. J Hand Surg Am 2006;31(8):1303-1307

13 Böhringer G, Schädel-Höpfner M, Petermann J, Gotzen L. A method for all-inside arthroscopic repair of Palmer 1B triangular fibrocartilage complex tears. Arthroscopy 2002;18(2):211-213

14 Corso SJ, Savoie FH, Geissler WB, Whipple TL, Jiminez W, Jenkins N. Arthroscopic repair of peripheral avulsions of the triangular fibrocartilage complex of the wrist: a multicenter study. Arthroscopy 1997;13(1):78-84

15 Chou KH, Sarris IK, Sotereanos DG. Suture anchor repair of ulnarsided triangular fibrocartilage complex tears. J Hand Surg $[\mathrm{Br}]$ 2003;28(6):546-550

16 de Araujo W, Poehling GG, Kuzma GR. New Tuohy needle technique for triangular fibrocartilage complex repair: preliminary studies. Arthroscopy 1996;12(6):699-703

17 del Piñal F, García-Bernal FJ, Cagigal L, Studer A, Regalado J, Thams C. A technique for arthroscopic all-inside suturing in the wrist. J Hand Surg Eur Vol 2010;35(6):475-479 
18 Haugstvedt JR, Husby T. Results of repair of peripheral tears in the triangular fibrocartilage complex using an arthroscopic suture technique. Scand J Plast Reconstr Surg Hand Surg 1999;33(4): 439-447

19 Hermansdorfer JD, Kleinman WB. Management of chronic peripheral tears of the triangular fibrocartilage complex. J Hand Surg Am 1991;16(2):340-346

20 Henry M. TFCC: a DRUJ perspective. In: del Pinal F, Mathoulin Ch, Nakamura T, eds. Arthroscopic Management of Ulnar Pain. Heidelberg, Germany: Springer-Verlag; 2012:121-135

21 Iwasaki N, Minami A. Arthroscopically assisted reattachment of avulsed triangular fibrocartilage complex to the fovea of the ulnar head. J Hand Surg Am 2009;34(7):1323-1326

22 Iwasaki N, Nishida K, Motomiya M, Funakoshi T, Minami A. Arthroscopic-assisted repair of avulsed triangular fibrocartilage complex to the fovea of the ulnar head: a 2- to 4-year follow-up study. Arthroscopy 2011;27(10):1371-1378

$23 \mathrm{Kim}$ B, Yoon HK, Nho JH, et al. Arthroscopically assisted reconstruction of triangular fibrocartilage complex foveal avulsion in the ulnar variance-positive patient. Arthroscopy 2013;29(11):1762-1768

24 Mathoulin C, Levadoux M, Martinache X. Intérêt thérapeutique de l'arthroscopie du poignet: a propos de 1000 cas. e-Mémoires de l'Académie Nationale de Chirurgie 2005;4(3):42-57

25 Millants P, De Smet L, Van Ransbeeck H. Outcome study of arthroscopic suturing of ulnar avulsions of the triangular fibrocartilage complex of the wrist. Chir Main 2002;21(5):298-300

26 Moritomo H, Masatomi T, Murase T, Miyake J, Okada K, Yoshikawa $\mathrm{H}$. Open repair of foveal avulsion of the triangular fibrocartilage complex and comparison by types of injury mechanism. J Hand Surg Am 2010;35(12):1955-1963

27 Nakamura T, Nakao Y, Ikegami H, Sato K, Takayama S. Open repair of the ulnar disruption of the triangular fibrocartilage complex with double three-dimensional mattress suturing technique. Tech Hand Up Extrem Surg 2004;8(2):116-123
28 Nakamura T, Sato K, Okazaki M, Toyama Y, Ikegami H. Repair of foveal detachment of the triangular fibrocartilage complex: open and arthroscopic transosseous techniques. Hand Clin 2011;27(3): 281-290

29 Pederzini LA, Tosi M, Prandini M, Botticella C. All-inside suture technique for Palmer class 1B triangular fibrocartilage repair. Arthroscopy 2007;23(10):1130.e1-1130.e4

30 Sennwald GR, Lauterburg M, Zdravkovic V. A new technique of reattachment after traumatic avulsion of the TFCC at its ulnar insertion. J Hand Surg [Br] 1995;20(2):178-184

31 Shinohara T, Tatebe M, Okui N, Yamamoto M, Kurimoto S, Hirata H. Arthroscopically assisted repair of triangular fibrocartilage complex foveal tears. J Hand Surg Am 2013;38(2):271-277

32 Skie MC, Mekhail AO, Deitrich DR, Ebraheim NE. Operative technique for inside-out repair of the triangular fibrocartilage complex. J Hand Surg Am 1997;22(5):814-817

33 Trumble TE, Gilbert M, Vedder N. Isolated tears of the triangular fibrocartilage: management by early arthroscopic repair. J Hand Surg Am 1997;22(1):57-65

34 Yao J, Dantuluri P, Osterman AL. A novel technique of all-inside arthroscopic triangular fibrocartilage complex repair. Arthroscopy 2007;23(12):1357.e1-1357.e4

35 Zachee B, De Smet L, Fabry G. Arthroscopic suturing of TFCC lesions. Arthroscopy 1993;9(2):242-243

36 Atzei A, Luchetti R, Sgarbossa A, Carità E, Llusà M. Set-up, portals, and normal exploration in wrist arthroscopy [in French]. Chir Main 2006;25(Suppl 1):S131-S144

37 del Piñal F, García-Bernal FJ, Pisani D, Regalado J, Ayala H, Studer A. Dry arthroscopy of the wrist: surgical technique. J Hand Surg Am 2007;32(1):119-123

38 Luchetti R, Atzei A, Cozzolino R, Fairplay T, Badur N. Comparison between open and arthroscopic-assisted foveal triangular fibrocartilage complex repair for post-traumatic distal radio-ulnar joint instability. J Hand Surg Eur Vol 2014;39(8):845-855 